\title{
Lipid Hydroperoxide
}

National Cancer Institute

\section{Source}

National Cancer Institute. Lipid Hydroperoxide. NCI Thesaurus. Code C124072.

Highly reactive, non-radical peroxols comprised of a fatty acid modified by a

hydroperoxide group $(-\mathrm{OOH})$ that are produced by lipid peroxidation. Lipid hydroperoxides are formed by sequential abstraction of allylic hydrogens from unsaturated fatty acids, which occurs in the presence of reactive oxygen species. These modified lipids can be further modified to create cytotoxic reactive aldehydes, such as malondialdehyde (MDA) and 4-hydroxy-2-nonenal (HNE). Lipid hydroperoxide levels can be measured to assess the extent of oxidative stress. 